Human \& Experimental Toxicology

\title{
Safety Assessment of Nicotinamide Riboside, a Form of Vitamin B3
}

\begin{tabular}{|c|c|}
\hline Journal: & Human and Experimental Toxicology \\
\hline Manuscript ID & Draft \\
\hline Manuscript Type: & Original Article \\
\hline Date Submitted by the Author: & $\mathrm{n} / \mathrm{a}$ \\
\hline Complete List of Authors: & $\begin{array}{l}\text { Conze, Dietrich; Spherix Consulting } \\
\text { Crespo-Barreto, Juan; Spherix Consulting } \\
\text { Kruger, Claire; Spherix Consulting }\end{array}$ \\
\hline Keyword: & $\begin{array}{l}\text { Niagen, Nicotinamide riboside, Nicotinamide, 90-day subchronic oral } \\
\text { toxicity, Genotoxicity }\end{array}$ \\
\hline Abstract: & $\begin{array}{l}\text { Nicotinamide riboside (NR) is a naturally occurring form of vitamin B3 } \\
\text { present in trace amounts in some foods. Like niacin, it has been shown to } \\
\text { be a NAD+ precursor in the biosynthesis of NAD+. The safety of Niagen, a } \\
\text { synthetic form of NR was determined using a bacterial reverse mutagenesis } \\
\text { assay (Ames), an in vitro chromosome aberration assay, an in vivo } \\
\text { micronucleus assay, and acute, } 14 \text {-day and } 90 \text {-day rat toxicology studies. } \\
\text { Niagen was not genotoxic. The oral LD50 was > } 5000 \mathrm{mg} / \mathrm{kg} \text {. Based on the } \\
\text { results of a 14-day study, a } 90 \text {-day study was performed comparing } \\
\text { Niagen at } 300,1000 \text { and } 3000 \mathrm{mg} / \mathrm{kg} / \text { day to an equimolar dose of } \\
\text { nicotinamide at } 1,260 \mathrm{mg} / \mathrm{kg} / \mathrm{day} \text { as a positive control. Results from the } \\
\text { study show that Niagen had a similar toxicity profile to nicotinamide at the } \\
\text { highest dose tested. Target organs of toxicity were liver, kidney, ovaries, } \\
\text { and testes. The lowest observed adverse effect level (LOAEL) for Niagen } \\
\text { was } 1000 \mathrm{mg} / \mathrm{kg} / \mathrm{day} \text {, and the no observed adverse effect level (NOAEL) } \\
\text { was } 300 \mathrm{mg} / \mathrm{kg} / \text { day. }\end{array}$ \\
\hline
\end{tabular}

\section{SCHOLARONE $^{\text {Ix }}$}

Manuscripts 
Safety Assessment of Nicotinamide Riboside

\title{
Safety Assessment of Nicotinamide Riboside, a Form of Vitamin B3
}

\author{
Dietrich B. Conze ${ }^{1,2}$, Juan Crespo-Barreto ${ }^{1,2}$, and Claire L. Kruger ${ }^{1,2,3}$ \\ ${ }^{1}$ Spherix Consulting, 11900 Parklawn Drive, Suite 200, Rockville, MD 20852 \\ ${ }^{2}$ These authors contributed equally to this manuscript \\ ${ }^{3}$ Corresponding author. Email: clairek@chromadex.com. Phone: 301-897-0611. Fax: 301- \\ $897-2567$
}


Safety Assessment of Nicotinamide Riboside

\begin{abstract}
Nicotinamide riboside (NR) is a naturally occurring form of vitamin B3 present in trace amounts in some foods. Like niacin, it has been shown to be a NAD+ precursor in the biosynthesis of NAD+. The safety of Niagen, a synthetic form of NR was determined using a bacterial reverse mutagenesis assay (Ames), an in vitro chromosome aberration assay, an in vivo micronucleus assay, and acute, 14-day and 90-day rat toxicology studies. Niagen was not genotoxic. The oral LD50 was $>5000 \mathrm{mg} / \mathrm{kg}$. Based on the results of a 14day study, a 90-day study was performed comparing Niagen at 300, 1000 and 3000 $\mathrm{mg} / \mathrm{kg} /$ day to an equimolar dose of nicotinamide at $1,260 \mathrm{mg} / \mathrm{kg} / \mathrm{day}$ as a positive control. Results from the study show that Niagen had a similar toxicity profile to nicotinamide at the highest dose tested. Target organs of toxicity were liver, kidney, ovaries, and testes. The lowest observed adverse effect level (LOAEL) for Niagen was $1000 \mathrm{mg} / \mathrm{kg} / \mathrm{day}$, and the no observed adverse effect level (NOAEL) was $300 \mathrm{mg} / \mathrm{kg} /$ day.
\end{abstract}

Keywords: Niagen; nicotinamide riboside; nicotinamide; 90-day subchronic oral toxicity; genotoxicity 
Safety Assessment of Nicotinamide Riboside

\title{
Introduction
}

\begin{abstract}
Niacin refers to nicotinamide, nicotinic acid and derivatives that exhibit the biologic activity of nicotinamide. Nicotinamide riboside (NR) is a single chemical moiety containing nicotinamide and ribose (Figure 1) and, because it is a precursor of nicotinamide adenine dinucleotide (NAD), is considered to be a form of vitamin B3 (reviewed in Chi and Sauve 2013; Penberthy and Kirkland, 2012; Bogan and Brenner, 2008). Although there is no evidence of adverse effects from naturally occurring niacin in foods, it is recognized that for nutrients, there is a daily level of intake or threshold above which the risk of adverse effects increase (defined as the tolerable upper intake level (UL)) (Institute of Medicine, 1998). A UL for NR has not been established by an authoritative or regulatory body. Therefore, to establish a UL for Niagen ${ }^{\mathrm{TM}}$, a synthetic form of NR, pivotal genotoxicity and toxicology studies were conducted.
\end{abstract}

Niacin is essential for the formation of the pyridine nucleotide coenzymes NAD and nicotinamide adenine dinucleotide phosphate (NADP). Both coenzymes function indispensably in oxidation-reduction reactions involved in glucose, fatty acid, ketone body, and amino acid catabolism. Once inside the cells, nicotinic acid is converted to NAD by the 
Safety Assessment of Nicotinamide Riboside

biosynthetic (Preiss-Handler) pathway and intracellular NAD glycosidases then release nicotinamide, which circulates to other tissues where it is absorbed and functions as a precursor for NAD and NADP formation (Preiss and Handler, 1957a; Preiss and Handler, 1957b; Preiss and Handler, 1958a; Preiss and Handler, 1958b; Collins and Chaykin, 1972). In contrast, nicotinamide and nicotinamide riboside are converted to NAD by way of nicotinamide mononucleotide in the salvage pathway (Rongvaux et al., 2002; Martin et al., 2001; Revollo et al., 2004; Bieganowski et al., 2004). When nicotinic acid and nicotinamide are in excess, both are methylated in the liver and excreted in the urine (Mrochek et al., 1976).

Because animal toxicology studies on nicotinic acid and nicotinamide are limited and do not comply with current standardized testing protocols, such as OECD (Unna, 1939; Handler and Dann, 1942; Chen et al., 1938; OECD SIDS, 2002), authoritative bodies have used the results clinical studies in which high doses of nicotinic acid and nicotinamide have been administered to derive the ULs. The most severe forms of toxicity after nicotinic acid ingestion are hepatotoxicity and glucose intolerance, and occur a doses greater than 500 $\mathrm{mg} /$ day. Flushing has also been reported, and generally occurs at doses greater than 50 
Safety Assessment of Nicotinamide Riboside $\mathrm{mg} /$ day. As a result, it is considered to be the most sensitive endpoint of nicotinic acid effects. In contrast, after ingestion of supplemental nicotinamide, no cases of flushing or glucose intolerance have been reported and only one case of hepatitis was reported following the ingestion of greater than $3 \mathrm{~g} /$ day for several days (Winter and Boyer, 1973).

Although nicotinamide does not appear to be associated with flushing, the IOM established a UL of $35 \mathrm{mg} /$ day for adults 19 years and older for both nicotinic acid and nicotinamide based on flushing because it is considered to be protective against potential adverse effects. The European Commission Scientific Committee on Food (SCF) established a UL of $10 \mathrm{mg}$ acid/day for nicotinic acid based on flushing (European Commission Scientific Committee on Food, 2002). In contrast, the SCF established a separate UL of $900 \mathrm{mg} /$ day for nicotinamide based on hepatic function in diabetic subjects ((European Commission Scientific Committee on Food, 2002). 
Safety Assessment of Nicotinamide Riboside

Materials and Methods

$\underline{\text { Bacterial Reverse Mutagenicity (Ames Assay) }}$

Bacterial reverse mutation assays were performed in compliance with the Organization for Economic Co-operation and Development (OECD) Principles of Good Laboratory Practices (GLP) and Guideline No. 471. Niagen ( $>99 \%$ nicotinamide riboside chloride; CAS No. 23111-00-4) was supplied by ChromaDex, Inc. 2-aminoanthracene, 2nitrofluorene, sodium azide, 9-aminoacridine, and 4-nitroquinoline- $N$-oxide were obtained from Sigma Aldrich Chemical Co., Inc. Aroclor 1254-induced rat liver S9 homogenate was obtained from Xenometrix AG. Salmonella typhimurium TA98, TA100, TA1535, and TA1537 were obtained from the National Collection of Type Cultures. Escherichia coli WP2 uvrA (pKM101) was obtained from Xenometrix.

The mutagenicity of Niagen was determined using the plate incorporation and preincubation methods. In the plate incorporation method, 50, 159, 501, 1582, and $5000 \mu \mathrm{g}$ Niagen was mixed with selective top agar containing $0.6-0.8 \%$ agar, $0.5 \% \mathrm{NaCl}$, the tester strains S. typhimurium TA98, TA100, TA1535 and TA1537 or Escherichia coli WP2 uvrA 
Safety Assessment of Nicotinamide Riboside pKM101, histidine and biotin or tryptophan (depending on the type stain used), with and without of a metabolic activation system (S9 mix; 5-30\% of the Aroclor 1254-induced rat liver S9 homogenate, NADP, glucose-6-phosphate, magnesium chloride, and potassium chloride) at $45-50^{\circ} \mathrm{C}$. The mixture was overlaid onto solidified Vogel-Bonner minimal E basal agar (Vogel and Bonner, 1956), and after the selective top agar solidified, the plates were incubated at $37^{\circ} \mathrm{C}$ for $67 \mathrm{hr}$. The plates were examined for the presence of a background lawn and precipitate, and the number of revertant colonies were counted manually. In a confirmatory assay, the tester strains $S$. typhimurium TA98, TA100, TA1535 and TA1537 or Escherichia coli WP2 uvrA pKM101, and 99, 265, 699, 1869, or $5000 \mu \mathrm{g}$ Niagen were preincubated at $37^{\circ} \mathrm{C}$ for $30 \mathrm{~min}$ in the presence or absence of the $\mathrm{S} 9 \mathrm{mix}$. Molten selective top agar containing $0.6-0.8 \%$ agar, $0.5 \% \mathrm{NaCl}$, and histidine and biotin or tryptophan (depending on the type stain used) was then added and the resulting mixture was plated and incubated at $37^{\circ} \mathrm{C}$ for $67 \mathrm{hr}$. The plates were examined for the presence of a background lawn and precipitate, and the number of revertant colonies were counted manually. 


\section{Safety Assessment of Nicotinamide Riboside}

Importantly, all experiments were performed in triplicate with vehicle and strainspecific positive controls. In the presence of the S9 mix, the positive controls for all strains was 2-aminoanthracene. In the absence of the S9 mix, the positive controls for strains TA98, TA100 and TA1535, TA1537, and WP2 uvrA pKM101 were 2-nitrofluorene, sodium azide, 9-aminoacridine, and 4-nitroquinoline- $N$-oxide, respectively. Niagen was considered cytotoxic if there was a 50\% reduction in the mean number of revertants per plate compared to the mean vehicle control and/or at least a moderate reduction in the background lawn. Niagen was considered mutagenic if there was a concentration-related increase in the number of revertants per plate in at least one tester strain over a minimum of two increasing concentrations of Niagen. In the case of the strains TA98, TA100, and WP2 uvrA pKM101 the result was considered positive if the mean number of revertants was equal to or greater than two times the number of revertants obtained with the negative control. In the case of the strains TA1535 and TA1537 the result was considered positive if the mean number of revertants was equal to or greater than three times the number of revertants obtained with the negative control. 
Safety Assessment of Nicotinamide Riboside

$\underline{\text { In vitro Chromosomal Aberration Assay }}$

In vitro chromosomal aberration assays were performed in compliance with the OECD Principles of GLP and Guideline No. 473. Niagen ( $>99 \%$ nicotinamide riboside chloride) was supplied by ChromaDex, Inc. Cyclophosphamide monohydrate and ethyl methanesulphonate were obtained from Sigma Aldrich Chemical Co., Inc. Aroclor 1254induced rat liver S9 homogenate was obtained from Xenometrix AG. Human peripheral blood lymphocytes (PBLs) were obtained from whole blood harvested from a healthy donor who was approximately 35 years-old, had no history of smoking or alcoholism, and had not received medication one month prior to the blood draw. The whole blood was cultured in RPMI 1640 medium containing 10\% fetal bovine serum, heparin, EDTA, amphotericin, penicillin, streptomycin, and phytohaemagglutinin (PHA) at $37^{\circ} \mathrm{C}$ and $5 \% \mathrm{CO}_{2}$ for 3 days per OCED Guideline 473 and International Conference on Harmonisation of Technical Requirements for Pharmaceuticals for Human Use (ICH)-harmonized guidances on genotoxicity testing of pharmaceuticals.

To determine the clastogenic activity of Niagen, the PHA-stimulated whole blood cultures were centrifuged and the resulting PBLs were resuspended in RPMI 1640 
Safety Assessment of Nicotinamide Riboside

containing $10 \%$ fetal bovine serum, amphotericin, penicillin, streptomycin, either vehicle (water), $1.25,2.5$, or $5 \mathrm{mg} / \mathrm{ml}$ of Niagen, or the appropriate positive control (cyclophosphamide monohydrate and ethyl methanesulphonate), supplemented with either phosphate buffered saline or the metabolic activation system (S9 mix; 10\% of the Aroclor 1254-induced rat liver S9 homogenate, 4mM NADP, $5 \mathrm{mM}$ glucose-6-phosphate, $8 \mathrm{mM}$ magnesium chloride, and $33 \mathrm{mM}$ potassium chloride). The mixtures were then incubated at $37^{\circ} \mathrm{C}$ and $5 \% \mathrm{CO}_{2}$ for 3 and 19 hours, at which point colchicine was added to the cultures to a final concentration of $2 \mu \mathrm{g} / \mathrm{ml}$. Three hours later, the cells were harvested by centrifugation at 800 to $1000 \mathrm{rpm}$ for approximately 10 minutes, resuspended in $0.56 \%$ pre-warmed potassium chloride, and incubated at room temperature for 25 to 30 minutes. The cell suspension was then centrifuged 800 to $1000 \mathrm{rpm}$ for approximately 10 minutes, the resulting supernatant was discarded, and the cellular pellet was resuspended and incubated in cold fixative (acetic acid: methanol (1:3)) at room temperature for 10 to 15 minutes. This process was repeated 3 additional times with one incubation at $4{ }^{\circ} \mathrm{C}$ for a minimum of 1 hour followed by two incubations at room temperature for 10 to 15 minutes. After the final incubation, the cells suspension was dropped onto clean, cold slides, which were then gently dried over a flame, stained with 5\% Giemsa, and scored for the presence $-10-$ 
Safety Assessment of Nicotinamide Riboside of metaphase cells and the presence of aberrations. To determine the mitotic index, which was used as an indicator of cytotoxicity, a minimum of 1000 cells were scored for each group and the total number of metaphases was divided by the number of cells counted. The quotient was then multiplied by 100 . To determine the types of aberrations (chromatid gaps, chromosomal gaps, chromosomal breaks, chromatid breaks, deletions, and fragments) a minimum of 300 metaphases containing $46+/-2$ centromere regions were counted and the number of cells containing one or more different types of aberrations were recorded. The data was the subjected to a one-tailed Fisher Exact test. Niagen was considered cytotoxic if there was a $45+/-5 \%$ reduction in the mitotic index compared to the vehicle control. Niagen was considered mutagenic if there was a concentration-related and statistically significant increase $(\mathrm{p}<0.05)$ in the number of chromosome aberrations.

$\underline{\text { In vivo Micronucleus Assay }}$

The in vivo micronucleus assay performed in compliance with the OECD Principles of GLP, OECD Guideline No. 474, and the recommendations of the Association for Assessment and Accreditation of Laboratory Animal Care International (AAALAC) and Committee for the Purpose of Control and Supervision of Experiments on Animals 
Safety Assessment of Nicotinamide Riboside

(CPCESA), Government of India. The rats were obtained from Harlan Laboratories.

Nutrilab Rodent Pellet feed was obtained from Provimi Animal Nutrition. Niagen ( $>99 \%$ nicotinamide riboside chloride; CAS No. 23111-00-4) was supplied by ChromaDex, Inc. Niagen ( $>99 \%$ nicotinamide riboside chloride) was supplied by ChromaDex, Inc. Cyclophosphamide was obtained from Sigma Aldrich Chemical Co., Inc.

All rats were housed at three rats per sex per cage, acclimatized for at least 5 days prior to treatment, and, except for the overnight fast prior to euthanasia on day 91, provided feed and water ad libitum throughout the study. Prior to dosing the rats were randomized by body weight to two groups ( $\mathrm{n}=6 / \mathrm{sex} /$ group). At dosing, a single dose of vehicle (water), 500,1000 , and $2000 \mathrm{mg} / \mathrm{kg}$ of Niagen or $40 \mathrm{mg} / \mathrm{kg}$ cyclophosphamide was administered by gavage at a rate of $10 \mathrm{ml} / \mathrm{kg}$ body weight. Twenty-four hours after dosing the vehicle, 500 mg/kg Niagen-, 1000 mg/kg Niagen-, 2000 mg/kg Niagen-, and 40 mg/kg cyclophosphamide-treated groups were euthanized by carbon dioxide asphyxiation. Fortyeight hours after dosing, an additional vehicle and $2000 \mathrm{mg} / \mathrm{kg}$ Niagen-treated group was also euthanized by carbon dioxide asphyxiation. The animals were observed for mortality at 
Safety Assessment of Nicotinamide Riboside

1 and 2 hours and then twice daily after dosing for 2 days. Clinical signs were monitored 1 and 2 hours after dosing and then once daily for 2 days.

Immediately after euthanisation, bone marrow was harvested from the femurs of each animal, and centrifuged. The centrifuged cell suspension was smeared on 2 slides, which were then air-dried, fixed in methanol, and stained using a May-Gruenwald and Giemsa solution. The test item was considered toxic if polychromatic erythrocyte/total erythrocyte ratio less than that in vehicle control group. Niagen were be considered mutagenic if, at least one of the treatment groups exhibited a statistically significant $(p<0.05)$ increase in the frequency of micronucleated immature erythrocytes when compared with the concurrent vehicle control.

\section{Acute Toxicity Study}

The acute toxicity study was performed in female Sprague Dawley SD rats in compliance with the OECD Principles of GLP, the Guidance for Industry, Single Dose Acute Toxicity Testing for Pharmaceuticals from the United States Food and Drug Administration, and the recommendations of the AAALAC and CPCESA, Government of 


\begin{abstract}
Safety Assessment of Nicotinamide Riboside
India. The rats were obtained from Harlan Laboratories. Nutrilab Rodent Pellet feed was obtained from Provimi Animal Nutrition. Niagen ( $>99 \%$ nicotinamide riboside chloride) was supplied by ChromaDex, Inc.
\end{abstract}

\begin{abstract}
All rats were housed two to three rats per sex per cage, acclimatized for at least 5 days prior to treatment, and, except for the overnight fast prior to dosing, provided feed and water ad libitum throughout the study. Prior to dosing the rats were randomized by body weight to two groups ( $\mathrm{n}=5 / \mathrm{sex} /$ group). At dosing, a single dose of vehicle (water) or 5000 $\mathrm{mg} / \mathrm{kg}$ of Niagen was administered by gavage at a rate of $10 \mathrm{ml} / \mathrm{kg}$ body weight. Over the course of the following 14 days all rats were monitored for morbidity, mortality, and visible clinical signs. Detailed clinical examinations were conducted on day 1, 8 and 15 and included evaluations of the skin, fur, eyes, mucous membranes, occurrence of secretions and excretions and autonomic activity, changes in gait, posture, response to handling, and presence of clonic or tonic movements, stereotypes, or bizarre behaviors. Body weights were recorded prior to treatment on day 1 and then on day 8 and 15. Food consumption for each cage was measured on day 8 and 15 and food consumption per rat was calculated by dividing the total food consumption during the interval per cage by the number of rats
\end{abstract}


Safety Assessment of Nicotinamide Riboside

multiplied by the number of days. On day 15, all animals were euthanized and examined for gross pathological changes. Analyses were conducted using two-tailed tests for a minimum significance level of 5\% comparing the Niagen and vehicle-treated group for each sex. All quantitative variables, like body weight, body weight gain, and food consumption were subjected to Student's t-test. Males and females were considered separately for each analyses and a $\mathrm{p}$ value of $<0.05$ was considered statistically significant.

\section{4-Day Repeat Dose Study}

A 14 day repeat-dose study was conducted in Sprague Dawley rats in compliance with the OECD Guideline 407, but for 14 days instead of 28 days, and the recommendations of the Association for Assessment and Accreditation of Laboratory Animal Care International (AAALAC) and Committee for the Purpose of Control and Supervision of Experiments on Animals (CPCESA), Government of India. The rats were obtained from Harlan Laboratories. Nutrilab Rodent Pellet feed was obtained from Provimi Animal Nutrition. Niagen ( $>99 \%$ nicotinamide riboside chloride) was supplied by ChromaDex, Inc. 
Safety Assessment of Nicotinamide Riboside

All rats were housed two to three rats per sex per cage, acclimatized for at least 5 days prior to treatment, and provided feed and water ad libitum throughout the study. Prior to dosing the rats were randomized to five groups ( $n=5 / \operatorname{sex} /$ group) according to body weight. During the 14-day treatment period, each group was gavaged daily with either vehicle (water) or $750,1500,2500$, or $5000 \mathrm{mg} / \mathrm{kg} /$ day of Niagen at a rate of $10 \mathrm{~mL} / \mathrm{kg}$ body weight. Dose formulation analyses showed that Niagen was completely soluble in water and the dose formulations were homogeneous and contained the targeted concentrations of nicotinamide riboside. Stability analyses showed that when Niagen was dissolved in water, nicotinamide riboside was stable up to 6 hours at room temperature and 7 days at $2-8{ }^{\circ} \mathrm{C}$. During the 14 day-treatment period all rats were monitored for morbidity, mortality, and visible clinical signs. Detailed clinical examinations were conducted on day 1, 8 and 15 and included evaluations of the skin, fur, eyes, mucous membranes, occurrence of secretions and excretions and autonomic activity, changes in gait, posture, response to handling, and presence of clonic or tonic movements, stereotypes, or bizarre behaviors. Body weights were recorded prior to treatment on day 1 and then on day 8 and 15. Food consumption for each cage was measured on day 8 and 15 and food consumption per rat was calculated by dividing the total food consumption during the interval per cage by the $-16-$ 
Safety Assessment of Nicotinamide Riboside number of rats multiplied by the number of days. On day 15, all animals were euthanized and examined for gross pathological changes. All analyses were conducted using two-tailed tests for a minimum significance level of 5\% comparing the Niagen and vehicle-treated group for each sex. All quantitative variables, like body weight, body weight gain, and food consumption were tested for normality and homogeneity of variances within the group before performing a one-factor ANOVA by treatment. When the data were found to be non-optimal, the data were log transformed prior to performing the ANOVA. Comparisons of the means differences between the Niagen- and vehicle-treated groups was performed using Dunnett's post-hoc test. When normality/homogeneity was significant, even after transformation, the data was subjected to the Kruskal-Wallis test followed by Dunn's posthoc test. Males and females were considered separately for each analyses and a $p$ value of $<0.05$ was considered statistically significant.

$\underline{\text { Subchronic Toxicity Study }}$

A 90-day oral subchronic toxicity study in Sprague Dawley rats was conducted in compliance with the OECD Principles of GLP, the OECD Guideline 408 for testing of chemicals, and the recommendations of the AAALAC and CPCESA, Government of India. $-17-$ 


\begin{abstract}
Safety Assessment of Nicotinamide Riboside
The rats were obtained from Harlan Laboratories. Nutrilab Rodent Pellet feed was obtained from Provimi Animal Nutrition. Niagen (>99\% nicotinamide riboside chloride) was supplied by ChromaDex, Inc. Nicotinamide ( $\geq 99.5 \%$; CAS No. 98-92-0) was obtained from Sigma Aldrich.
\end{abstract}

\begin{abstract}
All rats were housed two to three rats per sex per cage, acclimatized for at least 5 days prior to treatment, and, except for the overnight fast prior to euthanasia on day 91, provided feed and water ad libitum throughout the study. Prior to dosing the rats were randomized by body weight to 5 groups ( $n=10 /$ sex/group). During the 90 -day treatment period, each group was gavaged daily with either vehicle (water), 300, 1000, $3000 \mathrm{mg} / \mathrm{kg}$ of Niagen or $1260 \mathrm{mg} / \mathrm{kg} /$ day of nicotinamide, which is equivalent to $3000 \mathrm{mg} / \mathrm{kg} / \mathrm{day}$ of Niagen on a molar basis. Dose formulation analyses showed that both Niagen and nicotinamide were completely soluble in water and the dose formulations contained the targeted concentrations of nicotinamide riboside or nicotinamide. Stability analyses showed that when Niagen and nicotinamide were dissolved in water, both nicotinamide riboside and nicotinamide were stable up to 6 hours at room temperature and 7 days at $2-8{ }^{\circ} \mathrm{C}$. The parameters evaluated during the study were twice daily checks for mortality, daily
\end{abstract}




\section{Safety Assessment of Nicotinamide Riboside}

evaluations for clinical signs, weekly detailed clinical examinations, and weekly body weight and food consumption measurements. Ophthalmological examinations were performed prior to treatment and prior to sacrifice. On day 91, after urine was collected individually from all animals after an overnight fast, the animals were anesthetized, and blood was collected from the sublingual vein for hematology, coagulation, and clinical chemistry evaluations. Then the animals were euthanized by exsanguination under deep anesthesia and subjected to necropsy and gross pathological examination. Hematological parameters included differential leukocyte count (DLC), reticulocyte, leukocyte, erythrocyte, eosinophil, neutrophil, lymphocyte platelets, basophils, monocytes, and large unstained cell (LUC) counts, hemoglobin (Hgb), hematocrit, mean corpuscular volume (MCV), mean cell hemoglobin (MCH), mean hemoglobin concentration (MCHC), prothrombin time (PT), and activated partial thromboplastin time (APTT). Plasma clinical chemistry parameters included total protein, albumin, bile acids, aspartate aminotransferase (AST), alanine aminotransferase (ALT), gamma-glutamyl transpeptidase (GGTP), globulin, alkaline phosphatase (ALP), total cholesterol (TC), triglycerides, glucose, blood urea nitrogen (BUN), creatinine, inorganic phosphorous $(\mathrm{Pi})$, calcium $(\mathrm{Ca})$, magnesium $(\mathrm{Mg})$, sodium $(\mathrm{Na})$, potassium $(\mathrm{K})$, and chloride $(\mathrm{Cl})$ levels. The organs that were collected, 


\section{Safety Assessment of Nicotinamide Riboside}

weighed, and preserved included the adrenals, aorta, bone marrow smear, brain including medulla/pons, cerebellum and cerebrum, caecum, colon, duodenum, epididymides, esophagus, eyes with optic nerve, biceps femoris muscles, femur bone with joint gross lesions, heart, ileum with Peyer's patches, jejunum, kidneys, liver, lungs with main bronchi and bronchioles, mandibular lymph nodes, mesenteric lymph nodes, mammary gland, ovaries, oviducts, pancreas, pituitary, prostate, seminal vesicles and coagulating glands, rectum, salivary glands (mandibular, parotid and sublingual), sciatic nerve, skin (inguinal region), spinal cord at 3 levels - cervical, mid-thoracic and lumbar, spleen, sternum with marrow, stomach, testes, thymus, thyroid and parathyroid, tongue, trachea, urinary bladder, uterus with cervix, and vagina. The preserved tissues were processed and embedded in paraffin, sectioned and stained with Haematoxylin and Eosin. Histopathology was performed on all preserved organs of vehicle control, positive control nicotinamide and Niagen high dose. Liver, kidneys, thyroids, testes, epididymides (male), ovaries (female) and adrenals were examined at lower dose Niagen groups as treatment-related microscopic lesions were found in high dose group. Urine was analyzed for volume, osmotic pressure, specific gravity, $\mathrm{pH}$, and concentrations of glucose, bilirubin, ketone, blood, protein, urobilinogen, nitrate and the presence of leukocytes. All analyses were conducted using $-20-$ 
Safety Assessment of Nicotinamide Riboside two-tailed tests for a minimum significance level of 5\% comparing the Niagen and vehicletreated group for each sex. All quantitative variables, like body weight, body weight gain, and food consumption were tested for normality and homogeneity of variances within the group before performing a one-factor ANOVA by treatment. When the data were found to be non-optimal, the data were log transformed prior to performing the ANOVA. Comparisons of the means differences between the Niagen- and vehicle-treated groups was performed using Dunnett's post-hoc test. When normality/homogeneity was significant, even after transformation, the data was subjected to the Kruskal-Wallis test followed by Dunn's post-hoc test. Males and females were considered separately for each analyses and a $p$ value of $<0.05$ was considered statistically significant. 
Safety Assessment of Nicotinamide Riboside

Results

Bacterial Reverse Mutagenicity (Ames Assay)

Niagen was not cytotoxic at any of the doses used in this study (data not shown), and compared to the vehicle control, did not increase the number of revertant colonies in any of the frameshift or base-pair tester strains either when incubated in the presence or absence of the S9 mix, or using the plate incorporation or preincubation methods (Table 1). In contrast, all positive controls (2-Aminoanthracene 2-Nitrofluorene, 9-Aminoacridine, Sodium Azide, 4-Nitroquinoline- $N$-oxide) significantly increases in the number of revertant colonies $(\mathrm{p}<0.05)$, demonstrating both the sensitivity and validity of the assay. Therefore, Niagen was not mutagenic under the conditions used in the studies.

In vitro Chromosomal Aberration Assay

Niagen was not cytotoxic to ex vivo human peripheral blood lymphocytes at any of the concentrations used in the study as determined by the mitotic index (data not shown), and, compared to the vehicle control, did not increase the number of aberrant metaphases when incubated with or without S9 mix for 6 hours (Table 2). Moreover, the types of 


\section{Safety Assessment of Nicotinamide Riboside}

aberrations (chromatid gaps, chromosomal gaps, chromosomal breaks, chromatid breaks) detected in the vehicle- and Niagen-treated cells were similar. In contrast, the positive controls, cyclophosphamide and ethyl methansulphonate, significantly increased the number of aberrant metaphases $(p<0.05)$, characterized as chromatid gaps, chromosomal gaps, chromosomal breaks, chromatid breaks, deletions, and fragments, thus confirming the sensitivity and validity of the assay. Similar results were also found when the lymphocytes were incubated with increasing amounts of Niagen for 22 hours in the absence of the S9 mix (data not shown). Niagen was therefore not clastogenic under the conditions used in the study.

$\underline{\text { In vivo Micronucleus Assay }}$

No mortalities or clinical signs of toxicity were observed in any of the rats receiving Niagen. In addition, bone marrow analyses showed that compared to the negative control, the administration of 500,1000 , and $2000 \mathrm{mg} / \mathrm{kg}$ of did not result in cytotoxicity or increase the percentage of polychromatic erythrocytes at either 24 or 48 hours of administration (Table 3). In contrast, the positive control cyclophosphamide induced a statistically significant $(\mathrm{p}<0.05)$ increase in the percentage of polychromatic erythrocytes 
Safety Assessment of Nicotinamide Riboside

at 24 hours demonstrating both the sensitivity and validity of the assay. Therefore, Niagen was not genotoxic under the conditions used in this study.

\section{Acute Toxicity Study}

No mortalities were observed in males or females at the single tested dose of 5000 $\mathrm{mg} / \mathrm{kg}$ of Niagen. No clinical signs were observed in male and female rats. No statistically significant changes were observed in body weight in both sexes. Cumulative body weight gain (days 1-15) was significantly lower in female rats as compared to vehicle-treated group. Because change in day 15 body weight was minimal (-3\%), this was considered as treatment related but non-adverse. There were no statistically significant changes in food consumption in either sex. There were no gross pathological lesions observed in male and female rats. The LD50 for Niagen was determined to be greater than $5000 \mathrm{mg} / \mathrm{kg}$.

\section{4-day Toxicology Study}

Minimal reduction in mean body weight compared to the vehicle-treated group was observed in male rats at $2500 \mathrm{mg} / \mathrm{kg} / \mathrm{day}(7-8 \%$ reduction) and $5000 \mathrm{mg} / \mathrm{kg} / \mathrm{day}(8-9 \%$ reduction) on Days 8, 11, 14 and 15. A test article related decrease in overall (Day 1-14) 
Safety Assessment of Nicotinamide Riboside

feed consumption was observed at $5000 \mathrm{mg} / \mathrm{kg} / \mathrm{day}(8 \%)$ in male rats. No test item-related changes were observed in body weight and feed consumption in female rats. No gross pathological lesions were observed in male and female rats. Based on these results, the doses of 300,1000 and $3000 \mathrm{mg} / \mathrm{kg} /$ day were chosen for the 90 -day subchronic toxicity study in rats.

90-day Subchronic Toxicity Study

Mortality, body weight and feed consumption

No treatment related mortality or clinical signs were observed at any dose level in this study. Compared to vehicle-treated controls, a significant $(\mathrm{p}<0.05)$ treatment-related decrease in body weight ( $17 \%$ reduction) was noted in male rats at the high dose (3000 $\mathrm{mg} / \mathrm{kg} /$ day) of Niagen; a similar decrease in body weight was observed at an equimolar dose $(1260 \mathrm{mg} / \mathrm{kg} /$ day) of nicotinamide (Figure 2). A significant $(\mathrm{p}<0.05) 8-9 \%$ reduction body weight $(<10 \%)$ was observed at the 300 and $1000 \mathrm{mg} / \mathrm{kg} /$ day dose of Niagen in male rats. This decrease was $<10 \%$ and therefore not considered to be adverse. No statistically significant differences in body weights were seen in Niagen- or nicotinamide-treated 


\section{Safety Assessment of Nicotinamide Riboside}

females. In male rats, decreases in feed consumption were noted at $3000 \mathrm{mg} / \mathrm{kg} / \mathrm{day}$ of Niagen (9-14\%) and $1260 \mathrm{mg} / \mathrm{kg} /$ day of nicotinamide (9-17\%) throughout the treatment period. Decreases in feed consumption also occurred at $300 \mathrm{mg} / \mathrm{kg} /$ day on days 57-64 and at $1000 \mathrm{mg} / \mathrm{kg} /$ day on days 50-57. In female rats, decreases in feed consumption occurred at days 1-8 in the nicotinamide treated group and at days $15-22$ in the $3000 \mathrm{mg} / \mathrm{kg} / \mathrm{day}$ Niagen group.

Hematological, clinical chemistry and urinalysis tests

Similar treatment-related changes in hematology parameters were observed at the high dose Niagen- and nicotinamide-treated groups (Table 4). Statistically significant ( $<<$ $0.05)$ treatment related increases in white blood cells (WBC) and neutrophils occurred in both males and females. Statistically significant $(\mathrm{p}<0.05)$ increases in monocytes were also noted in females treated with $3000 \mathrm{mg} / \mathrm{kg} /$ day of Niagen and $1260 \mathrm{mg} / \mathrm{kg} /$ day of nicotinamide. At $1000 \mathrm{mg} / \mathrm{kg} /$ day of Niagen, significant increases in WBC and neutrophils were observed in males and females, respectively. There were no significant changes in hematological parameters at male or female rats treated with $300 \mathrm{mg} / \mathrm{kg} / \mathrm{d}$ of Niagen. Importantly, the significant effects were not associated with any inflammatory changes in $-26-$ 
Safety Assessment of Nicotinamide Riboside

any of the organs examined. All other changes in hematology parameters, including those determined to be statistically significant, were considered to be due to normal biological variation, and not due to the administration of the test item.

Niagen produced statistically significant $(\mathrm{p}<0.05)$ increases at $3000 \mathrm{mg} / \mathrm{kg} /$ day in ALT, ALP, GGT, triglycerides and bile acids; the effects on ALT and triglycerides were significant at $1000 \mathrm{mg} / \mathrm{kg}$ /day of Niagen in females (Table 5). Comparable effects on clinical chemistries were seen in the nicotinamide-treated group. A minimal but statistically significant decrease in sodium in females and chloride in males and females was noted at $3000 \mathrm{mg} / \mathrm{kg} /$ day of Niagen. Similar results were seen in the nicotinamidetreated group. Significant reductions $(\mathrm{p}<0.05)$ in sodium were also observed in males and females treated with $1000 \mathrm{mg} / \mathrm{kg} /$ day. All other changes in clinical chemistry parameters were considered incidental as the magnitude of change minimal and were considered to be due to normal biological variation.

Urinalysis showed increased urine volume in both nicotinamide and high dose Niagen- treated males. This effect was considered a treatment-related effect, which may have been correlated with microscopic changes in the adrenals. Urine $\mathrm{pH}$ was decreased in $-27-$ 


\section{Safety Assessment of Nicotinamide Riboside}

males and females in the $3000 \mathrm{mg} / \mathrm{kg} /$ day Niagen high dose males $(20 \%)$ and females (13\%), but not the males and females treated with nicotinamide. The slightly acidic $\mathrm{pH}$ may have been due to the excretion of test item and an acidic metabolite.

\section{Gross Pathology}

Bilateral small-size testes was observed in $3000 \mathrm{mg} / \mathrm{kg} /$ day Niagen- and nicotinamide-treated males was considered to be treatment-related and associated with degeneration/atrophy of the seminiferous tubules.

One single incidence of auxiliary region subcutaneous nodule microscopically associated with adenocarcinoma of mammary gland was observed in one Niagen high dose male. This neoplastic change was considered an incidental tumor of spontaneous origin as it is reported to occur naturally in young Sprague Dawley male rats (Ikezaki et al., 2011).

\footnotetext{
All other single or low incidences of gross pathologic findings observed in different groups were considered incidental and not related to test item as they were randomly distributed in different groups and were not dose-dependent.
} 


\section{Organ Weights}

At $3000 \mathrm{mg} / \mathrm{kg} / \mathrm{day}$, there were statistically significant reductions in absolute organ weights of brain, spleen, testes, epididymides, prostate, thyroid/parathyroid, pituitary and heart in males; brain and pituitary absolute organ weights were reduced and liver and kidney absolute organ weights were increased in females. In the nicotinamide-treated group, there were statistically significant reductions in absolute organ weights of brain, spleen, testes, epididymis, prostate, thyroid/parathyroid, pituitary, thymus and heart in males; brain and pituitary absolute organ weights were reduced and liver and ovary weights were increased in females. At $1000 \mathrm{mg} / \mathrm{kg} /$ day, there were statistically significant reductions in absolute organ weights of thyroid/parathyroid, pituitary and heart in males; no effects on absolute organ weights were seen in females. At $300 \mathrm{mg} / \mathrm{kg} / \mathrm{day}$, there were statistically significant reductions in absolute organ weights of brain and heart in males; no effects on absolute organ weights were seen in females. Relative organ weight changes in the $3000 \mathrm{mg} / \mathrm{kg} /$ day Niagen-treated rats were similar to those of animals ingesting an equimolar dose of nicotinamide (Table 6). Treatment-related organ weight changes were observed in liver, kidneys, testes, epididymides and ovaries in $3000 \mathrm{mg} / \mathrm{kg} /$ day Niagen- and 


\begin{abstract}
Safety Assessment of Nicotinamide Riboside nicotinamide-treated groups. At $1000 \mathrm{mg} / \mathrm{kg} /$ day of Niagen, increases in liver and kidney weights were statistically significant. All other relative to body weight organ weight changes which reached statistical significance were likely secondary to decrease in terminal body weight and/or random biological variation and not considered treatment related.
\end{abstract}

Relative to brain weight, at $3000 \mathrm{mg} / \mathrm{kg} / \mathrm{day}$, there were statistically significant reductions in heart, epididymides, prostate and thyroid/parathyroid in males; liver, heart, ovaries and kidney were increased in females. In the nicotinamide-treated group, there were statistically significant reductions in weights of spleen, epididymides, testes and heart in males; liver weight was increased. At $1000 \mathrm{mg} / \mathrm{kg} /$ day there were no changes in organ weights relative to brain weight in males and a statistically significant increase in liver weight in females. No changes in organ weights relative to brain weights were seen in either males or females treated with $300 \mathrm{mg} / \mathrm{kg} /$ day of Niagen.

\title{
Histopathological Findings
}

Treatment-related histopathological changes were observed in liver, thyroid, kidneys, testes, epididymides, ovaries and adrenals in both the $3000 \mathrm{mg} / \mathrm{kg} /$ day Niagen- 
Safety Assessment of Nicotinamide Riboside

treated and nicotinamide-treated groups. All other single or low incidences of microscopic findings observed were considered incidental and not related to test item as they were randomly distributed among groups s. Importantly, the treatment-related histopathological changes noted in Niagen at the high dose were similar to the findings observed in the equimolar nicotinamide group.

In the livers of $3000 \mathrm{mg} / \mathrm{kg} /$ day Niagen-treated and nicotinamide-treated and treated males and females centrilobular hepatocellular hypertrophy, characterized by enlarged hepatocytes containing granular eosinophilic cytoplasm, follicular cell hypertrophy, characterized by enlarged follicular epithelium which contained pale eosinophilic cytoplasm and small clear vacuoles, and hepatocyte single cell necrosis were reported and considered a treatment-related adverse change. In the kidneys, chronic progressive nephropathy characterized by presence of foci or areas of basophilic tubules, with or without simple tubular hyperplasia, hyaline casts, atrophic tubules, dilated tubule, focal glomerular sclerosis/atrophy and mononuclear cell infiltration was seen.

In male rats, both $3000 \mathrm{mg} / \mathrm{kg}$ /day Niagen-treated and nicotinamide-treated rats exhibited degeneration/atrophy of seminiferous tubules characterized by the presence of $-31-$ 


\begin{abstract}
Safety Assessment of Nicotinamide Riboside
some tubules containing degenerating germ cells. Some tubules were also depleted of all germ cells and lined only by Sertoli cells and while others were partially depleted of germ cells. Degenerating tubules contained multinucleated germ cells, spermatid head retention, Sertoli cell cytoplasmic vacuolation and disorganization of germ cells. Reduced sperm and cell debris in epididymal lumen in nicotinamide and Niagen high dose males were considered treatment-related effects.
\end{abstract}

In female rats of the $3000 \mathrm{mg} / \mathrm{kg} /$ day Niagen-treated and nicotinamide-treated groups, hypertrophy of corpora lutea was seen. The affected ovaries contained large sized corpora lutea and lightly eosinophilic cytoplasm of the enlarged luteal cells.

\begin{abstract}
In male and female rats of the $3000 \mathrm{mg} / \mathrm{kg} /$ day Niagen-treated and nicotinamidetreated groups, hypertrophy of the zona glomerulosa of the adrenal cortex was considered a treatment-related non-adverse change. Hypertrophy of zona glomerulosa was characterized by increased thickness of zona glomerulosa layer and cytoplasm of hypertophic cells was lightly eosinophilic.
\end{abstract}


Safety Assessment of Nicotinamide Riboside

\section{Discussion}

The safety of a synthetic form of NR was determined in an Ames assay, in vitro chromosome aberration assay, in vivo micronucleus assay and acute, 14-day and 90-day rat toxicology studies to derive a UL.

The determination of a UL for any vitamin is based on well-established principles of risk assessment and relies on data concerning adverse health effects from excessive nutrient intakes in epidemiologic studies, clinical trials, and experimental studies. Several factors associated with these various data sources influence the derivation of a UL. Among the most important of these factors are the intake at which adverse effects occur (the LOAEL) and the maximum level of intake, which is always less than the LOAEL, at which no adverse health effects are observed (the NOAEL) (IOM, 1998).

There are no animal toxicology studies on nicotinic acid and nicotinamide that comply with current standardized testing protocols from which to derive a UL. Therefore, authoritative bodies have used the results clinical studies in which high doses of nicotinic acid and nicotinamide have been administered. A UL of $35 \mathrm{mg} /$ day for adults was 
Safety Assessment of Nicotinamide Riboside

established for both nicotinic acid and nicotinamide by the IOM (1998) based on the flushing in humans as the critical adverse effect. However, nicotinamide does not induce flushing when either given as an intravenous injection or orally at high-doses to patients with diabetes (SCF, 2002), and therefore, it is currently thought that the flushing effects are related to the presence of the carboxyl group on the pyridine nucleus of nicotinic acid (Figure 1)(European Commission Scientific Committee on Food, 2002). NR also lacks this carboxyl group and thus may not produce the flushing response that is associated with high nicotinic acid intakes (Figure 1). Furthermore, in vitro studies have shown that NR does not induce the GPR109A-mediated calcium flux, which is believed to be required for nicotinic acid-induced flushing (Benyo et al., 2005; Canto et al., 2012). It is therefore appropriate to derive the ULs for nicotinamide and NR based on endpoints other than flushing.

Consistent with this, the European Commission and UK Expert Group on Vitamins and Minerals derived independent ULs for nicotinamide and nicotinic acid. The SCF (2002) set the UL for nicotinamide of $900 \mathrm{mg} /$ day based on long-term studies in patients with Type 1 diabetes mellitus, at dosages of 2-3 $\mathrm{g}$ of nicotinamide per day whereas the UK 
Safety Assessment of Nicotinamide Riboside

Expert Group on Vitamins and Minerals set that UL at $500 \mathrm{mg} /$ day (UK Expert Group on Vitamins and Minerals, 2003) based on human studies where large doses (up to 3000 $\mathrm{mg}$ /day for periods of up to 3 years) appeared to be well tolerated.

NR is not genotoxic. In addition, NR is thought to exhibit the same toxicity profile as nicotinamide because evidence from a single dose pharmacokinetic study in humans suggests that it is metabolized in a manner similar to nicotinamide (unpublished results). Because there are no publicly available 90 -day studies on either nicotinamide or NR, a toxicology study was completed where 300,1000 , or $3000 \mathrm{mg} / \mathrm{kg}$ body weight/day of NR, or $1260 \mathrm{mg} / \mathrm{kg}$ body weight/day of nicotinamide, which is equivalent to $3000 \mathrm{mg} / \mathrm{kg} / \mathrm{day}$ dose of NR on a molar basis, was administered to rats over the course of 90 days. Adverse effects at $3000 \mathrm{mg} / \mathrm{kg}$ body weight/day of NR included treatment-related adverse effects in liver, kidneys, testes, epididymides and ovaries. These effects included increases in clinical chemistry parameters related to hepatocyte damage (ALT, ALP, and GGT) and a corresponding increase in liver weight, centrilobular hepatocellular hypertrophy and single cell necrosis. In addition, thyroid follicular cell hypertrophy and increased kidney weight with exacerbation of chronic progressive nephropathy were observed. Statistically 


\begin{abstract}
Safety Assessment of Nicotinamide Riboside
significant but minor reductions in sodium and chloride were seen and microscopically associated with hypertrophy of zona glomerulosa in adrenals at $3000 \mathrm{mg} / \mathrm{kg}$. Importantly, these effects also occurred in the nicotinamide groups with a similar magnitude.
\end{abstract}

Niagen administration at $1000 \mathrm{mg} / \mathrm{kg} /$ day dose level resulted in treatment-related organ weight changes in liver and kidney and increases in neutrophils, ALT and triglycerides, which were statistically significant in female rats only. Although these changes were considered adverse, based their dose-dependent responsiveness, the increases in ALT and triglycerides occurred only in one gender and were below the 2-fold increase that is typically used as the cut-off for a biologically significant effect in the absence of histological results (Hall et al., 2012). The kidney weight increases at this dose also occurred in the absence of corresponding histopathology. Therefore, the liver and kidney effects at $1000 \mathrm{mg} / \mathrm{kg} /$ day were considered to be treatment-related, but mild and potentially adaptive in nature due to prolonged exposure to this form of niacin. There were no treatment related adverse effects noted at $300 \mathrm{mg} / \mathrm{kg} /$ day, although there was a slight decrease $(8 \%$ ) in overall body weight (Day 90) at $300 \mathrm{mg} / \mathrm{kg} / \mathrm{day}$, which was considered 


\section{Acknowledgements}

We would like to thank Syngene International Limited for performing all studies and WR Grace for manufacturing Niagen.

\section{Declaration of Conflicting Interests}

Dietrich Conze, Juan Crespo-Barreto, and Claire Kruger are employed by ChromaDex, Inc. 
Safety Assessment of Nicotinamide Riboside

\section{Funding}

This research received no specific grant from any funding agency in the public, commercial, or not-for-profit sectors. 
Safety Assessment of Nicotinamide Riboside

\section{References}

1. Benyo, Z., Gille, A., Kero, J., Csiky, M., Suchankova, M.C., Nusing, R.M., Moers, A., Pfeffer, K., and Offermanns, S. (2005). GPR109A (PUMA-G/HM74A) mediates nicotinic acid-induced flushing. J Clin Invest 115, 3634-3640.

2. Bieganowski $\mathrm{P}$ and Brenner C. Discoveries of nicotinamide riboside as a nutrient and conserved NRK genes establish a Preiss-Handler independent route to NAD + in fungi and humans. Cell. 2004 May 14;117(4):495-502.

3. Bogan, K. L., and Brenner, C. (2008). Nicotinic acid, nicotinamide, and nicotinamide riboside: a molecular evaluation of NAD+ precursor vitamins in human nutrition. Annual Review of Nutrition 28, 115-130.

4. Canto, C., Houtkooper, R. H., Pirinen, E., Youn, D. Y., Oosterveer, M. H., Cen, Y., Fernandez-Marcos, P. J., Yamamoto, H., Andreux, P. A., Cettour-Rose, P., et al. (2012). The $\mathrm{NAD}(+)$ precursor nicotinamide riboside enhances oxidative metabolism and protects against high-fat diet-induced obesity. Cell metabolism 15, 838-847.

5. Chen, K.K., Rose, C.L., and Robbins, E.B. (1938). Toxicity of nicotinic acid. Experimental Biology and Medicine 38, 241-245. 
Safety Assessment of Nicotinamide Riboside

6. Collins, P.B., and Chaykin, S. (1972). The management of nicotinamide and nicotinic acid in the mouse. J Biol Chem 247, 778-783.

7. Chi, Y., and Sauve, A. A. (2013). Nicotinamide riboside, a trace nutrient in foods, is a Vitamin $B_{3}$ with effects on energy metabolism and neuroprotection. Current opinion in clinical nutrition and metabolic care 16, 657-661.

8. Expert Group on Vitamins and Minerals (2003). Risk Assessment (Nicotinic Acid and Nicotinamide).

9. European Commission Scientific Committee on Food. (2002). The Tolerable Upper Intake Levels of Nicotinic Acid and Nicotinamide (Niacin). SCF/CS/NUT/UPPLEV 39.

10. Hall AP, Elcombe CR, Foster JR, Harada T, Kaufmann W, Knippel A, Küttler K, Malarkey DE, Maronpot RR, Nishikawa A, Nolte T, Schulte A, Strauss V, York MJ. Liver hypertrophy: a review of adaptive (adverse and non-adverse) changes-conclusions from the 3rd International ESTP Expert Workshop. Toxicol Pathol. 2012 Oct;40(7):971-94. Epub 2012 Jun 21.

11. Handler, P., and Dann, W.J. (1942). The inhibition of rat growth by nicotinamide. Journal of Biological Chemistry 146, 357-368. 
Safety Assessment of Nicotinamide Riboside

12. Ikezaki S, Takagi M, Tamura K. Natural occurrence of neoplastic lesions in young sprague-dawley rats. J Toxicol Pathol. 2011 Mar;24(1):37-40. doi: 10.1293/tox.24.37. Epub 2011 Mar 31.

13. Institute of Medicine (1998). Niacin. In Dietary Reference Intakes for Thiamine, Riboflavin, Niacin, Vitamin B6, Folate, Vitamin B12, Pantothenic Acid, Biotin, and Choline, ed. (Washington, DC: National Academy Press), pp. 123-149.

14. Martin, P.R., Shea, R.J., and Mulks, M.H. (2001). Identification of a plasmid-encoded gene from Haemophilus ducreyi which confers NAD independence. J Bacteriol 183, $1168-1174$.

15. Mrochek, J.E., Jolley, R.L., Young, D.S., and Turner, W.J. (1976). Metabolic response of humans to ingestion of nicotinic acid and nicotinamide. Clin Chem 22, $1821-1827$.

16. OECD Guideline for Testing Of Chemicals 471, Bacterial Reverse Mutation Test, 21 st July 1997.

17. OECD SIDS, (2002). 3-Pyridinecarboxamide (nicotinamide) - SIDS Initial Assessment Report for SIAM 15. 
Safety Assessment of Nicotinamide Riboside

18. Penberthy, W.T., and Kirkland, J.B. (2012). Niacin. In Present Knowledge in Nutrition, Erdman, J.W., I.A. Macdonald, and S.H. Zeisel, eds. (Ames: WileyBlackwell), pp. 293-306.

19. Preiss, J., and Handler, P. (1957a). Intermediates in the Synthesis of Diphosphopyridine Nucleotide from Nicotinic Acid. Journal of the American Chemical Society 79, 4246-4247.

20. Preiss, J., and Handler, P. (1957b). Synthesis of Diphosphopyridine Nucleotide from Nicotinic Acid by Human Erythrocytes In Vitro. Journal of the American Chemical Society $79,1514-1515$.

21. Preiss, J., and Handler, P. (1958a). Biosynthesis of diphosphopyridine nucleotide. I. Identification of intermediates. J Biol Chem 233, 488-492.

22. Preiss, J., and Handler, P. (1958b). Biosynthesis of diphosphopyridine nucleotide. II. Enzymatic aspects. J Biol Chem 233, 493-500.

23. Revollo, J.R., Grimm, A.A., and Imai, S. (2004). The NAD biosynthesis pathway mediated by nicotinamide phosphoribosyltransferase regulates Sir2 activity in mammalian cells. J Biol Chem 279, 50754-50763. 


\section{Safety Assessment of Nicotinamide Riboside}

24. Rongvaux, A., Shea, R.J., Mulks, M.H., Gigot, D., Urbain, J., Leo, O., and Andris, F. (2002). Pre-B-cell colony-enhancing factor, whose expression is up-regulated in activated lymphocytes, is a nicotinamide phosphoribosyltransferase, a cytosolic enzyme involved in NAD biosynthesis. Eur J Immunol 32, 3225-3234.

25. Unna, K. (1939). Studies on the toxicity and pharmacology of nicotinic acid. J Pharmacol Exp Ther 65, 95-103.

26. Vogel, H.J., and Bonner, D.M. (1956). Acetylornithinase of Escherichia coli: partial purification and some properties. J Biol Chem 218, 97-106.

27. Winter, S.L., and Boyer, J.L. (1973). Hepatic toxicity from large doses of vitamin B3 (nicotinamide). N Engl J Med 289, 1180-1182. 
Safety Assessment of Nicotinamide Riboside

\section{Figures and Tables}
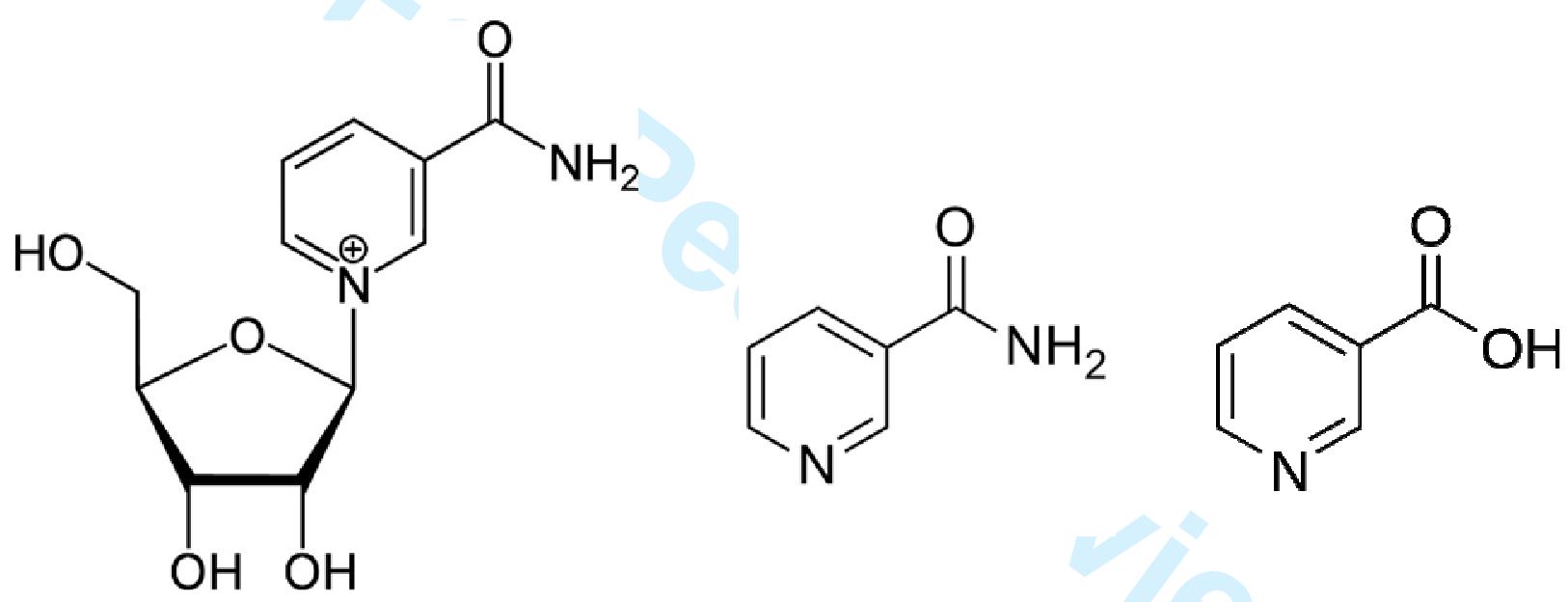

Figure 1. Structure of nicotinamide riboside (left), nicotinamide (middle), and nicotinic acid (right).

$-44-$ 
Safety Assessment of Nicotinamide Riboside
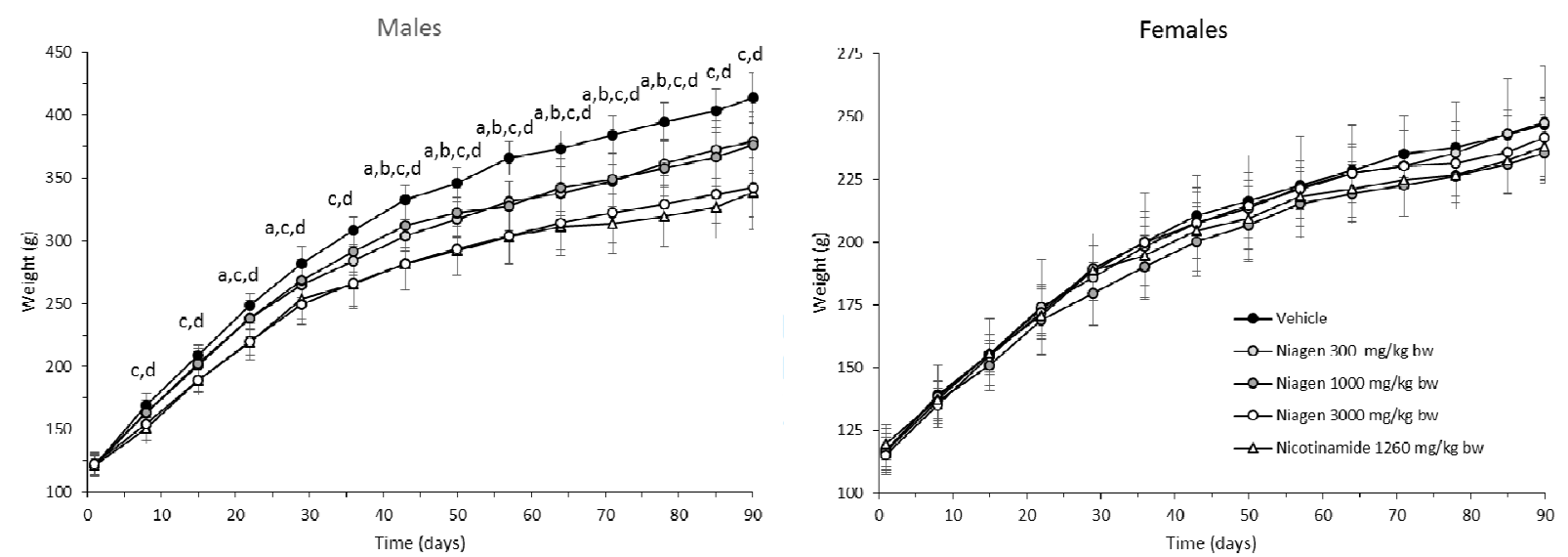

Figure 2. Body weights of male and female rats treated with vehicle control, nicotinamide riboside or nicotinamide for 90-days. "a", "b", "c", and "d" denote significant $(\mathrm{p}<0.05)$ differences between rats treated with 300,1000 , and $3000 \mathrm{mg} / \mathrm{kg} /$ day of Niagen, or $1260 \mathrm{mg} / \mathrm{kg} /$ day of nicotinamide, respectively, and rats treated with the vehicle control. 
Safety Assessment of Nicotinamide Riboside

\begin{tabular}{|c|c|c|c|c|c|c|c|}
\hline \multicolumn{8}{|c|}{ Table 1. Ames Assay Results } \\
\hline & \multirow[b]{3}{*}{ Test Item } & \multirow{3}{*}{$\begin{array}{c}\text { Test } \\
\text { Concentrations } \\
(\mu \mathrm{g} / \mathrm{plate})\end{array}$} & \multicolumn{5}{|c|}{ Mean colonies/plate } \\
\hline & & & \multicolumn{2}{|c|}{ Frameshift types } & \multicolumn{2}{|c|}{ Base-pair types } & $\begin{array}{c}\text { Frameshift } \\
\text { and base-pair } \\
\text { types }\end{array}$ \\
\hline & & & TA98 & TA1537 & TA1535 & $\begin{array}{l}\text { WP2uvrA } \\
\text { pKM101 }\end{array}$ & TA100 \\
\hline \multicolumn{8}{|c|}{ Plate Incorporation method } \\
\hline \multirow[t]{8}{*}{ With S9 Mix } & Water & - & $22 \pm 3$ & $8 \pm 2$ & $10 \pm 3$ & $149 \pm 3$ & $101 \pm 1$ \\
\hline & Niagen & 50 & $27 \pm 6$ & $8 \pm 2$ & $12 \pm 3$ & $153 \pm 3$ & $108 \pm 6$ \\
\hline & & 159 & $23 \pm 4$ & $11 \pm 3$ & $8 \pm 2$ & $152 \pm 3$ & $103 \pm 3$ \\
\hline & & 501 & $22 \pm 5$ & $8 \pm 3$ & $10 \pm 2$ & $142 \pm 4$ & $104 \pm 3$ \\
\hline & & 1582 & $26 \pm 1$ & $9 \pm 3$ & $12 \pm 2$ & $144 \pm 8$ & $103 \pm 1$ \\
\hline & & 5000 & $24 \pm 4$ & $10 \pm 8$ & $11 \pm 6$ & $144 \pm 6$ & $104 \pm 2$ \\
\hline & 2-Aminoanthracene & 4 & $597 \pm 14$ & $83 \pm 3$ & $108 \pm 4$ & - & $883 \pm 12$ \\
\hline & 2-Aminoanthracene & 30 & - & - & - & $736 \pm 6$ & - \\
\hline \multirow[t]{6}{*}{ Without S9 Mix } & Water & - & $21 \pm 4$ & $8 \pm 4$ & $12 \pm 4$ & $119 \pm 6$ & $104 \pm 2$ \\
\hline & Niagen & 50 & $27 \pm 4$ & $9 \pm 2$ & $14 \pm 5$ & $121 \pm 16$ & $106 \pm 3$ \\
\hline & & 159 & $23 \pm 6$ & $9 \pm 5$ & $14 \pm 3$ & $116 \pm 6$ & $108 \pm 5$ \\
\hline & & 501 & $27 \pm 4$ & $7 \pm 2$ & $17 \pm 5$ & $111 \pm 10$ & $111 \pm 6$ \\
\hline & & 1582 & $26 \pm 3$ & $7 \pm 4$ & $18 \pm 7$ & $122 \pm 14$ & $121 \pm 15$ \\
\hline & & 5000 & $27 \pm 4$ & $8 \pm 3$ & $18 \pm 4$ & $122 \pm 17$ & $121 \pm 3$ \\
\hline
\end{tabular}

http://mc.manuscriptcentral.com/het 
Safety Assessment of Nicotinamide Riboside

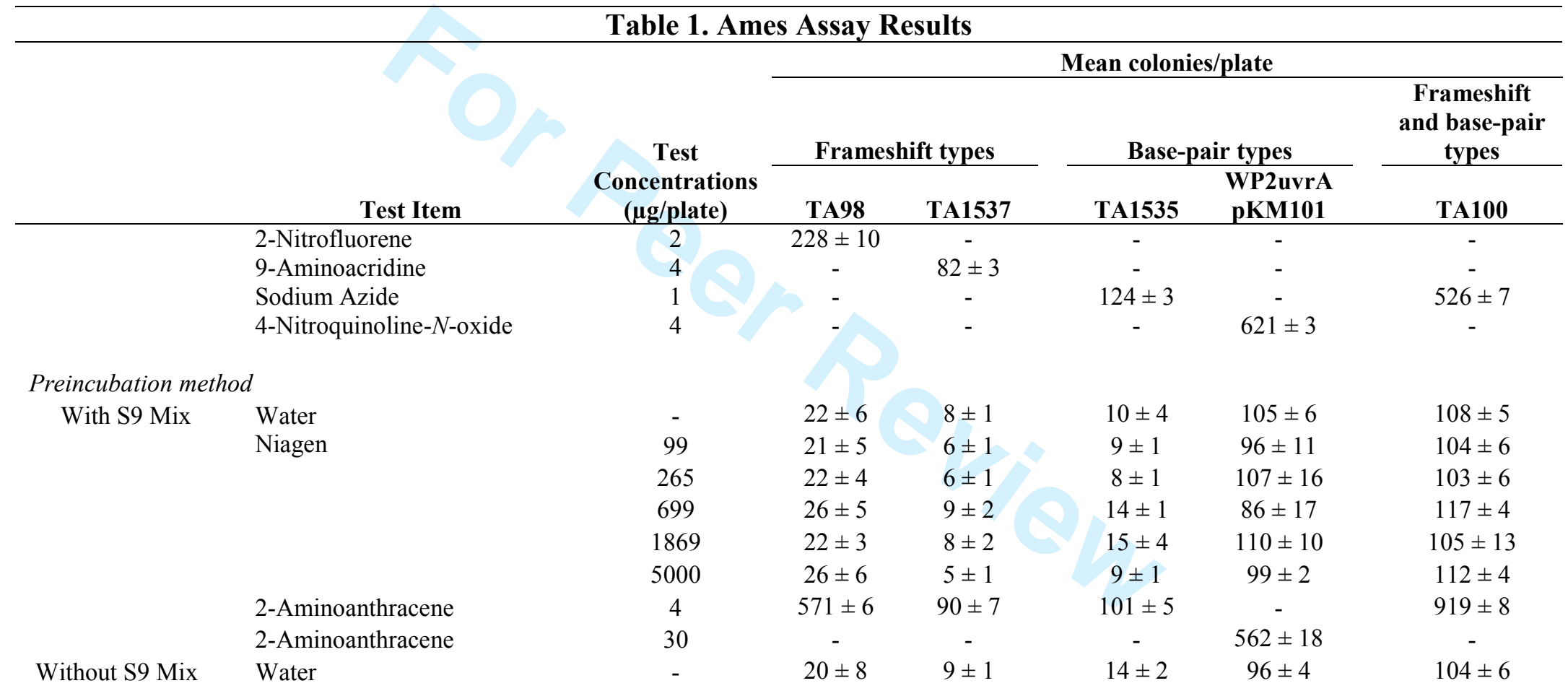

$-47-$ 
Safety Assessment of Nicotinamide Riboside

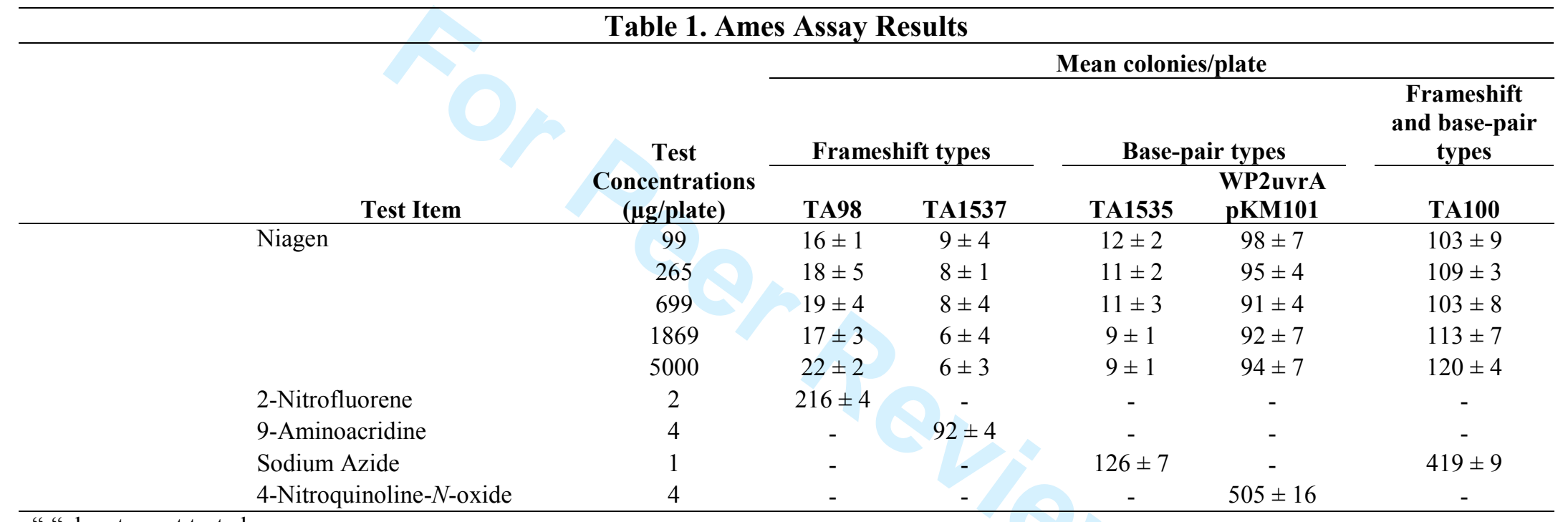

"_" denotes not tested

Values are mean +/- standard deviation

$-48-$ 
Safety Assessment of Nicotinamide Riboside

\begin{tabular}{|c|c|c|c|c|c|c|}
\hline \multicolumn{7}{|c|}{ Table 2: Summary of Results- In vivo Micronucleus test } \\
\hline \multirow[b]{2}{*}{ Parameters } & \multicolumn{6}{|c|}{ Dose $(\mathrm{mg} / \mathrm{kg}$ bw) } \\
\hline & $\begin{array}{c}\text { Vehicle Control } \\
0\end{array}$ & $\begin{array}{c}\text { Niagen } \\
500\end{array}$ & $\begin{array}{c}\text { Niagen } \\
1000\end{array}$ & $\begin{array}{c}\text { Niagen } \\
2000 \text { (a) }\end{array}$ & $\begin{array}{c}\text { Niagen } \\
2000 \text { (b) }\end{array}$ & $\begin{array}{c}\text { Positive Control } \\
40\end{array}$ \\
\hline \multicolumn{7}{|l|}{ Males } \\
\hline Sampling time (hours) & 24 & 24 & 24 & 24 & 48 & 24 \\
\hline PCE's with micronuclei (\%) & 0.00 & 0.00 & 0.00 & 0.00 & 0.00 & $0.28 *$ \\
\hline Range & $0-1$ & 0 & $0-1$ & 0 & 0 & 8-15 \\
\hline Mean $\mathrm{P} / \mathrm{E}$ ratio & 0.34 & 0.37 & 0.39 & 0.38 & 0.39 & 0.40 \\
\hline Females & & & & & & \\
\hline Sampling time (hours) & 24 & 24 & 24 & 24 & 48 & 24 \\
\hline PCE's with micronuclei (\%) & 0.00 & 0.00 & 0.00 & 0.00 & 0.00 & $0.27 *$ \\
\hline Range & 0 & 0 & 0 & 0 & 0 & $7-13$ \\
\hline Mean $\mathrm{P} / \mathrm{E}$ ratio & 0.40 & 0.43 & 0.44 & 0.44 & 0.41 & 0.40 \\
\hline
\end{tabular}

$-49-$ 
Safety Assessment of Nicotinamide Riboside

Table 3: Summary of Significant Changes-Hematological Parameters Day 91

\begin{tabular}{|c|c|c|c|c|c|}
\hline \multicolumn{6}{|c|}{ Doses (mg/kg/day) } \\
\hline Parameters & $\begin{array}{c}\text { Vehicle } \\
0\end{array}$ & $\begin{array}{c}\text { Niagen } \\
\mathbf{3 0 0}\end{array}$ & $\begin{array}{c}\text { Niagen } \\
1000\end{array}$ & $\begin{array}{c}\text { Niagen } \\
3000\end{array}$ & $\begin{array}{c}\text { Nicotinamide } \\
1_{1260^{\wedge}}\end{array}$ \\
\hline \multicolumn{6}{|l|}{ Males } \\
\hline White Blood Cells & $8.47 \pm 1.97$ & $9.16 \pm 1.54$ & $11.11 \pm 1.77^{*}$ & $13.51 \pm 2.64^{*}$ & $14.11 \pm 1.66^{*}$ \\
\hline Total Neutrophils & $2.12 \pm 0.7$ & $2.30 \pm 1.67$ & $2.86 \pm 1.20$ & $5.76 \pm 1.47 *$ & $6.02 \pm 0.7 *$ \\
\hline Total Monocytes & $0.28 \pm 0.09$ & $0.33 \pm 0.30$ & $0.40 \pm 0.19$ & $0.42 \pm 0.09$ & $0.45 \pm 0.12$ \\
\hline \multicolumn{6}{|l|}{ Females } \\
\hline White Blood Cells & $6.25 \pm 1.20$ & $6.23 \pm 1.84$ & $7.40 \pm 1.01$ & $10.38 \pm 1.51 *$ & $9.99 \pm 2.64 *$ \\
\hline Total Neutrophils & $0.93 \pm 0.31$ & $1.11 \pm 0.57$ & $1.63 \pm 0.29 *$ & $3.36 \pm 1.19 *$ & $4.13 \pm 1.56 *$ \\
\hline Total Monocytes & $0.16 \pm 0.07$ & $0.15 \pm 0.05$ & $0.15 \pm 0.04$ & $0.32 \pm 0.08 *$ & $0.33 \pm 0.21 *$ \\
\hline $\begin{array}{r}\text { Abbreviations } \\
\text { Values repres } \\
\text { *Significantly } \\
\wedge \text { : eq }\end{array}$ & $\begin{array}{l} \pm \mathrm{SD} \\
\text { e vehicle cor } \\
\text { Niagen } 300\end{array}$ & $\mathrm{up}_{/ \mathrm{d}}$ at $p<0.05$. & & & \\
\hline
\end{tabular}


Safety Assessment of Nicotinamide Riboside

\begin{tabular}{|c|c|c|c|c|c|}
\hline \multicolumn{6}{|c|}{ Table 4: Summary of Significant Changes-Clinical Chemistries Day 91} \\
\hline \multicolumn{6}{|c|}{ Doses (mg/kg/day) } \\
\hline Parameters & $\begin{array}{c}\text { Vehicle } \\
0\end{array}$ & $\begin{array}{c}\text { Niagen } \\
\mathbf{3 0 0}\end{array}$ & $\begin{array}{c}\text { Niagen } \\
1000\end{array}$ & $\begin{array}{c}\text { Niagen } \\
\mathbf{3 0 0 0}\end{array}$ & $\begin{array}{c}\text { Nicotinamide } \\
1260^{\wedge}\end{array}$ \\
\hline \multicolumn{6}{|l|}{ Males } \\
\hline $\operatorname{ALT}(\mathrm{U} / \mathrm{L})$ & $85.23 \pm 26.11$ & $75.94 \pm 12.20$ & $106.17 \pm 45.34$ & $159.35 \pm 27.56 *$ & $152.11 \pm 25.50 *$ \\
\hline AST (U/L) & $122.18 \pm 27.34$ & $116.10 \pm 13.94$ & $138.23 \pm 49.03$ & $132.93 \pm 23.63$ & $139.47 \pm 26.99$ \\
\hline ALP (U/L) & $99.85 \pm 22.69$ & $120.94 \pm 16.68$ & $110.96 \pm 18.67$ & $131.63 \pm 19.73 *$ & $139.48 \pm 24.88 *$ \\
\hline GGT (U/L) & $3.09 \pm 0.90$ & $4.13 \pm 1.22$ & $3.47 \pm 1.20$ & $3.82 \pm 1.22$ & $3.81 \pm 1.16$ \\
\hline Trig (mg/dL) & $49.84 \pm 18.96$ & $59.30 \pm 29.48$ & $69.65 \pm 29.91$ & $128.34 \pm 47.88^{*}$ & $98.57 \pm 34.72 *$ \\
\hline Sodium (mmol/L) & $139.15 \pm 3.37$ & $141.03 \pm 6.05$ & $137.99 \pm 3.52$ & $137.1 \pm 4.7$ & $135.88 \pm 1.9$ \\
\hline Chloride (mmol/L) & $101.55 \pm 2.66$ & $102.13 \pm 4.41$ & $99.21 \pm 1.3$ & $97.34 \pm 3.24 *$ & $97.52 \pm 1.25 *$ \\
\hline \multicolumn{6}{|l|}{ Females } \\
\hline ALT (U/L) & $56.09 \pm 15.54$ & $56.34 \pm 10.47$ & $76.50 \pm 20.42 *$ & $121.81 \pm 22.35^{*}$ & $125.22 \pm 30.29 *$ \\
\hline AST (U/L) & $101.02 \pm 18.67$ & $106.1 \pm 18.52$ & $126.69 \pm 35.51$ & $126.27 \pm 33.09$ & $126.31 \pm 16.24^{*}$ \\
\hline $\operatorname{ALP}(\mathrm{U} / \mathrm{L})$ & $60.79 \pm 5.16$ & $69.66 \pm 11.23$ & $85.96 \pm 22.06$ & $105.10 \pm 26.99 *$ & $114.41 \pm 30.91 *$ \\
\hline GGT (U/L) & $3.15 \pm 1.17$ & $3.9 \pm 0.92$ & $3.83 \pm 1.42$ & $5.01 \pm 1.36 *$ & $5.46 \pm 1.75^{*}$ \\
\hline
\end{tabular}


Safety Assessment of Nicotinamide Riboside

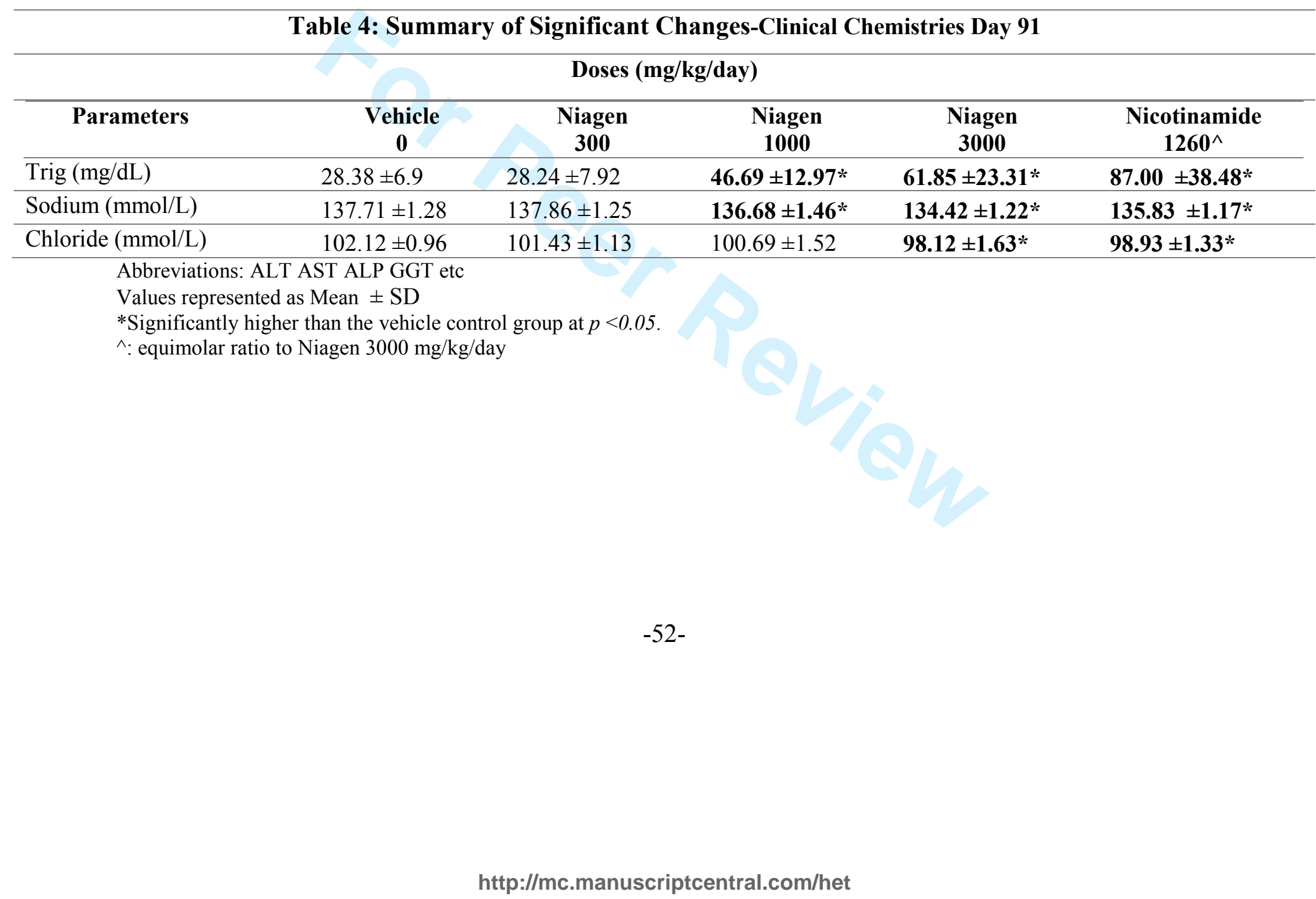


Safety Assessment of Nicotinamide Riboside

\begin{tabular}{|c|c|c|c|c|c|}
\hline \multicolumn{6}{|c|}{ Table 5: Summary of Significant Changes-Organ Weight Ratio Relative to Body Weight At Day 91} \\
\hline \multirow[b]{2}{*}{ Parameters } & \multicolumn{3}{|c|}{ Dose $(\mathrm{mg} / \mathrm{kg} / \mathrm{day})$} & \multirow[b]{2}{*}{$\begin{array}{c}\text { Niagen } \\
3000\end{array}$} & \multirow[b]{2}{*}{$\begin{array}{c}\text { Nicotinamide } \\
1260^{\wedge}\end{array}$} \\
\hline & $\begin{array}{c}\text { Vehicle } \\
0\end{array}$ & $\begin{array}{l}\text { Niagen } \\
300\end{array}$ & $\begin{array}{c}\text { Niagen } \\
1000\end{array}$ & & \\
\hline Males & & & & & \\
\hline Terminal Body Wt. & $395.7 \pm 18.36$ & $363.56 \pm 23.22 *$ & $354.23 \pm 21.85 *$ & $317.21 \pm 25.8 *$ & 312.87 $\pm 26.74 *$ \\
\hline \multicolumn{6}{|l|}{ Organ/body weight } \\
\hline Liver & $2.958 \pm 0.143$ & $3.013 \pm 0.163$ & $3.200 \pm 0.18 *$ & $3.600 \pm 0.272 *$ & $3.703 \pm 0.116 *$ \\
\hline Kidneys & $0.715 \pm 0.047$ & $0.701 \pm 0.033$ & $\mathbf{0 . 7 7 7} \pm \mathbf{0 . 0 2} *$ & $0.876 \pm 0.063 *$ & $0.833 \pm 0.086 *$ \\
\hline Brain & $0.507 \pm 0.027$ & $0.526 \pm 0.038$ & $0.550 \pm 0.024 *$ & $0.572 \pm 0.042$ & $0.583 \pm 0.043 *$ \\
\hline Heart & $0.368 \pm 0.019$ & $0.356 \pm 0.018$ & $0.373 \pm 0.015$ & $0.384 \pm 0.02$ & $0.387 \pm 0.015$ \\
\hline Thymus & $0.068 \pm 0.011$ & $0.076 \pm 0.015$ & $0.086 \pm 0.014 *$ & $0.07 \pm 0.009$ & $0.068 \pm 0.008$ \\
\hline Adrenals & $0.012 \pm 0.001$ & $0.013 \pm 0.001$ & $0.014 \pm 0.001 *$ & $0.014 \pm 0.001 *$ & $0.015 \pm 0.001 *$ \\
\hline Females & & & $(\infty)$ & & \\
\hline Terminal Body Wt. & $232.29 \pm 8.1$ & $234.43 \pm 23.28$ & $219.51 \pm 9.92$ & $216.19 \pm 14.75$ & $215.61 \pm 11.16$ * \\
\hline \multicolumn{6}{|l|}{ Organ/body weight } \\
\hline Liver & $2.902 \pm 0.191$ & $3.003 \pm 0.327$ & $3.295 \pm 0.181 *$ & $4.046 \pm 0.174 *$ & $4.465 \pm 0.239 *$ \\
\hline
\end{tabular}

$-53-$ 
Safety Assessment of Nicotinamide Riboside

\begin{tabular}{|c|c|c|c|c|c|}
\hline \multicolumn{6}{|c|}{ Table 5: Summary of Significant Changes-Organ Weight Ratio Relative to Body Weight At Day 91} \\
\hline \multicolumn{6}{|c|}{ Dose $(\mathrm{mg} / \mathrm{kg} / \mathrm{day})$} \\
\hline Parameters & $\begin{array}{c}\text { Vehicle } \\
0\end{array}$ & $\begin{array}{c}\text { Niagen } \\
\mathbf{3 0 0}\end{array}$ & $\begin{array}{c}\text { Niagen } \\
1000\end{array}$ & $\begin{array}{c}\text { Niagen } \\
\mathbf{3 0 0 0}\end{array}$ & $\begin{array}{c}\text { Nicotinamide } \\
1260^{\wedge}\end{array}$ \\
\hline Kidneys & $0.676 \pm 0.053$ & $0.645 \pm 0.06$ & $0.678 \pm 0.058$ & $0.822 \pm 0.044 *$ & $0.766 * \pm 0.019$ \\
\hline Brain & $0.78 \pm 0.034$ & $0.788 \pm 0.055$ & $0.801 \pm 0.047$ & $0.798 \pm 0.041$ & $0.781 \pm 0.031$ \\
\hline Heart & $0.392 \pm 0.016$ & $0.393 \pm 0.015$ & $0.398 \pm 0.022$ & $0.433 \pm 0.032 *$ & $0.424 * \pm 0.029$ \\
\hline Thymus & $0.09 \pm 0.011$ & $0.097 \pm 0.024$ & $0.093 \pm 0.013$ & $0.087 \pm 0.014$ & $0.084 \pm 0.017$ \\
\hline Adrenals & $0.027 \pm 0.003$ & $0.028 \pm 0.003$ & $0.027 \pm 0.002$ & $0.029 \pm 0.004$ & $0.027 \pm 0.003$ \\
\hline Ovaries & $0.036 \pm 0.005$ & $0.037 \pm 0.005$ & $0.036 \pm 0.006$ & $0.045 \pm 0.008 *$ & $0.049 \pm 0.007 *$ \\
\hline
\end{tabular}

